\title{
THE THERAPEUTIC ATTITUDE IN TREATING LESIONS OF THE ARTERIOPATHIC PREDOMINANT DIABETIC FOOT
}

\author{
F. Bobirca ${ }^{1}$, E. Catrina ${ }^{1}$, O. Mihalache ${ }^{1}$, Simona Carniciü ${ }^{2}$, T. Pătrașcu ${ }^{1}$ \\ 1"Dr. I. Cantacuzino” Clinical Hospital, "Carol Davila” University of Medicine and Pharmacy \\ 2"N. C. Paulescu" National Institute for Diabetes, Nutrition and Metabolic Diseases \\ Bucharest, Romania
}

\begin{abstract}
Background. The diabetic foot is a complex pathologic entity that includes modifications occurred at the foot level, which can be found either at the layer of nerve, skin, bone or muscle tissue, either on blood vessels. A special issue is that of tardiness in coming to the doctor in such patients that have severe lesions, for the most part requiring the performance of a minor or major amputation, long periods of treatment and permanent social assistance. Data from the medical literature are showing that early and adequate care given to diabetic foot lesions may prevent amputation of the injured limb in as many as $80 \%$ of cases. The aim of the research is to clarify the surgical indication for the arteriopathic predominant diabetic foot lesions.

Material and methods. A retrospective analysis was performed on a number of 320 predominantly arteriopathic diabetic patients, from the case records of the Cantacuzino Hospital, "I.Juvara" Surgery Clinic, taking into account all aspects related to the compensation and evolution of the diabetic disease, as well as the study of diabetic foot lesions, the surgical therapeutic attitude and the results obtained.

Results. An examination of the obtained results reveals the fact that a large number of amputations were performed in $93 \%$ of cases (298 patients), of which minor amputations (transphalangeal, transmetatarsal, toe/s and foot) accounted for $69 \%$ of cases (220 patients), and major amputations (lower leg and upper leg) accounted for $24 \%$ of cases (78 patients). For the rest of the patients, excisional debridements were performed, accompanied by complementary therapy, namely done by applying local topicals. The post surgery evolution has been unfavourable in $7 \%$ ( 22 patients) of the transphalangeal amputation cases, in $16 \%$ (51 patients) of the toe amputation patients (transmetatarsal toe/s) and in $4 \%$ (13 patients) of the transmetatarsal foot amputation cases, the surgical solution has consisted of a major amputation.

Conclusions. The arteriopathic terrain of the diabetic patient, evaluated with the ankle-brachial index, is a major factor in determining the evolution towards amputation.
\end{abstract}

Key words: diabetes, gangrene, diabetic foot

\section{Introduction}

Diabetes mellitus is the endocrine disease with the highest rate of occurrence, in approximately $10 \%$ of the general population. Diabetes represents a major public health issue due to the alarmingly high number of new cases registered on an annual basis, by its negative impact on the quality and duration of life, but also due to the high costs incurred by treating a diabetic patient.[1]

Address for correspondence: Florin-Teodor Bobirca, MD

"Carol Davila" University of Medicine and Pharmacy, Bucharest, Romania, e-mail: btf278@yahoo.com 
The diabetic foot occurs as a chronic complication of diabetes and is represented by all the modifications that occur at the level of the foot as a result of the diabetic disease presence and its complications. The diabetic foot is a consequence of modifications appeared at the vascular, nerve, skin and bone level. From the etiopathogenic point of view there can be distinguished the arteriopathic predominant diabetic foot and the neuropathic predominant diabetic foot, both conditions showing modifications in the two categories of lesions. [2] To the two classical causes, arteriopathy and neuro-pathy, other apparently minor factors are added, such as habitual trauma caused by shoes, skin mycosis of the nails, limited mobility of the joints and the degree of physical exercise of the patient. [3]

Gangrene (fig. 1) is the most severe complication of the diabetic foot and is characterized by the presence of necrosis lesions, generated by vascular and nervous damage, to which an infectious factor is added [4]. This is responsible for a large number of major amputations, the mortality rate of patients with diabetic gangrene, measured according to several studies, and is ranging between 0.5 and 6 $\%$. $[4,5,6]$

The diagnostic of arteriopathic diabetic foot is established on the grounds of a clinical examination: cold, cyanotic teguments, lack of pilosism, absence of perception of the peripheral pulse, a local moderate-severe algic syndrome (which is one of the main causes for the patient coming to see a specialist).[2] The diagnosis is made also on the grounds of paraclinical examinations: ankle-brachial index, that offers us details with a sensitivity of $90 \%$ and a specificity of $98 \%$, with regard to the detection of hemodynamic significant arterial stenoses; Doppler ultrasound; arteriography; but also the nuclear magnetic resonance, used mainly in such cases in which the arteriography does not yield conclusive results. [2] (fig. 2)

The microbial flora which is most frequently encountered in patients with diabetic foot disease is a polymorphous flora, represented by:[2]

Treatment of the diabetic foot comprises a multidisciplinary approach that includes: the general practitioner, the diabetologist, the surgeon, the neurologist, the orthopaedist, the ophthalmologist, the specialized nurses, in order to secure an early detection of lesions, of disorders of the sight and ability to walk, of peripheral sensitivity alteration, because social seclusion and

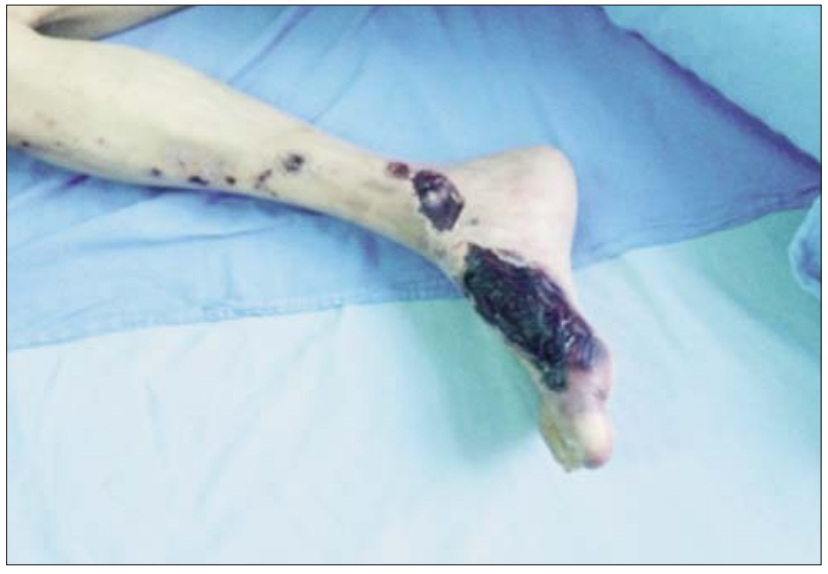

Figure 1 - Ischemic necrosis of the foot and parcellary lower leg

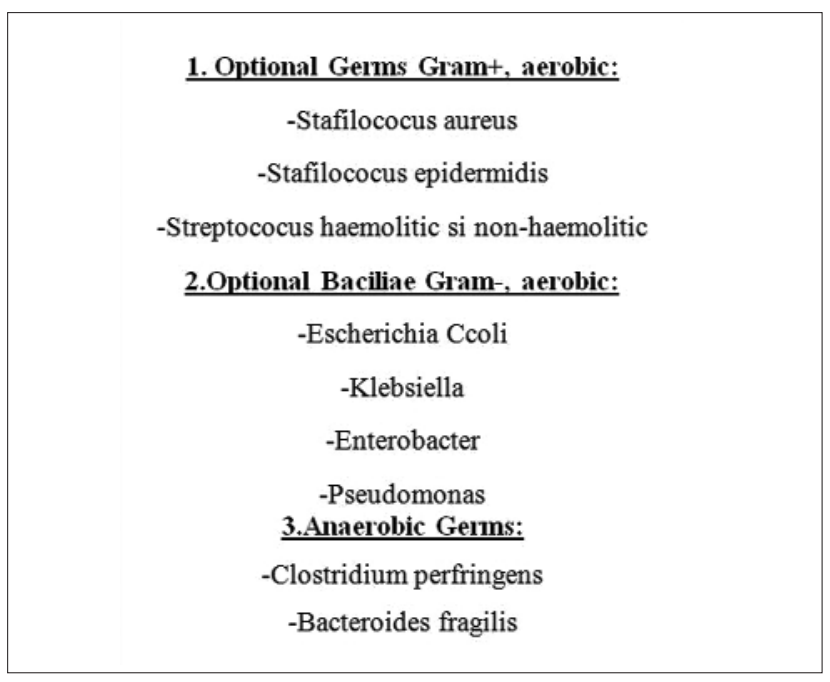

Figure 2 - Flora from diabetic foot infections (1)

lack of compliance by some patients represent major risk factors for the diabetic foot lesions and implicitly for amputations.[5]

Treatment of the arteriopathic predominant diabetic foot leads to a decrease in the number of invalidating surgery by means of introducing complementary methods of treatment, meant to support the conventional surgery (debridements, transphalangeal and transmetatarsal amputations, of the toe/s, foot, lower leg and upper leg amputations), such as: local bandaging with specific indications[7], varying according to the type of the resulted wound, negative pressure therapy[7,8,9], hyperbaric oxygen therapy and surgical revascularization methods.

The aim of the study is to establish the proper surgical indication for arteriopathic predominant diabetic foot lesions. 


\section{Material and Methods}

A retrospective analysis was done on a number of 320 patients with arteriopathic predominant diabetic foot that were admitted throughout the time interval January 2012 - December 2012, at the „I. Juvara” Surgery Clinic within the Dr.I.Cantacuzino Clinical Hospital.

The patients were selected on the grounds of lacking a perception of peripheral pulse in the pedis artery.

The study shows data regarding the evolution of the diabetic disease: onset and type of diabetes, degree of metabolic compensation, other complications thereof (retinopathy, nephropathy, cardiovascular diseases), as well as information about the moment of occurrence of the diabetic foot lesions, the type of surgical intervention performed, reinterventions, associated treatments, studying the implications by means of correlating these parameters.

Using this parameter the study analyses the appropriate surgical intervention for each type of lesion, the postoperative outcome by means of level of amputation, the number of reinterventions and the period until a major amputation was done. The results of using vascular surgery on arteriopathic diabetic foot correlated with the anklebrachial index and the type of lesion, were analysed also.

\section{Results}

The percentage distribution of patients according to gender has revealed a larger presence of arteriopathy diabetic foot lesions in male patients $(72 \%$, 230 patients), compared to $28 \%$ (90 patients) of the female patients, thus confirming data from literature and mainly being caused by a large number of alcohol and tobacco consumers among male patients.

As far as the treatment of diabetes is concerned, the data reveal a metabolic compensation by means of oral anti-diabetic medication in $66 \%$ of the analyzed cases (211 patients), and by means of insulin-therapy in 34\% of cases (109 patients) and the oral treatment was able to provide a balance of the diabetic disease which was lower than the one provided by administering insulin, a result which is supported by the medical literature [2, 10].

By studying the patients in the lot we were able to find that in most cases the lesions have occurred after more than 10 years of evolution of the diabetic disease, in $76 \%$ of cases (243 patients) associated cardiovascular diseases were present (arterial hypertension, cardiac insufficiency, sequellary myocardial infarction, sequellary stroke), in 43\% of cases (137 patients) a diabetic nephropathy was present. In $53 \%$ of the analyzed cases (170 patients) there was a septic condition of the patients upon admission to the hospital, which represented an additional factor of seriousness, adding to an already severe clinical picture of patients affected by a long evolution of the diabetic disease and its complications [11].

The peripheral pulse in the posterior tibiae artery was imperceptible in $88 \%$ of the studied patients (281 patients) and in the pedis artery the pulse was absent in $100 \%$ of cases.

As far as surgical intervention was concerned, in $46 \%$ of cases (147 patients) there was a transmetatarsal amputation of toe/s performed for ischemic gangrene lesions (fig. 3), in $16 \%$ of cases (51 patients) a reintervention was required as a result of an unfavourable evolution, for the occurrence of ischemia phenomena at the wound, fact that required the performance of a major amputation (lower leg, upper leg). In 16\% (51 patients) the surgical intervention consisted of a transmetatarsal foot amputation, with an unfavourable evolution in $4 \%$ of cases (13 patients) that required either the performance of a transtarsial amputation, or of a major amputation.

A transphalangeal resection has been the surgical intervention performed on $12 \%$ of cases ( 38 patients), patients that upon admission were showing parcellary necrosis at toe level, however

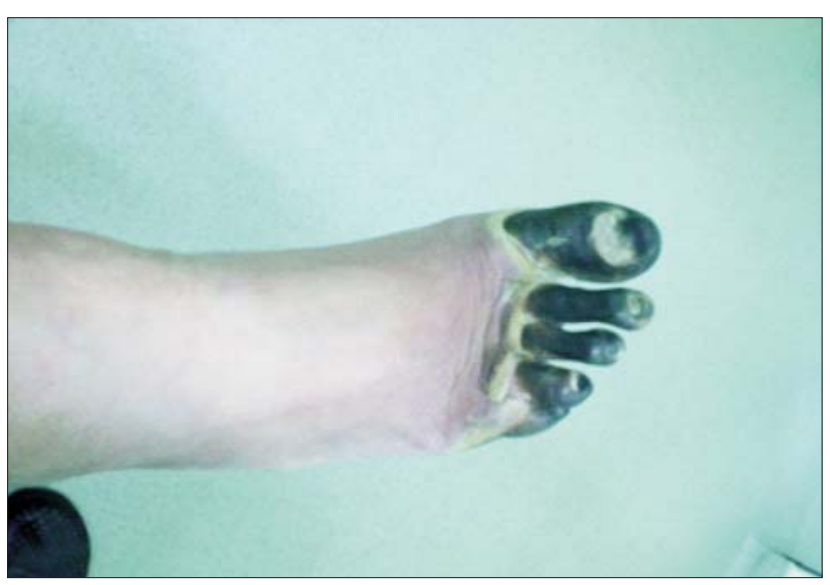

Figure 3 - Ischemic gangrene toes I-V 
with reintervention in $7 \%$ of cases ( 22 patients), performing a toe amputation, for the post-surgery wound necrosis.

In $17 \%$ (54 patients) of the patients belonging to the analyzed lot excisional debridements were performed, followed by a recommendation to apply special bandages locally, separately meant for each type of wound, with an unfavourable evolution in $7 \%$ of case ( 22 patients) where reintervention was needed by means of a surgical solution consisting of a transphalangeal resection in 3\% of cases (10 patients) and a transmetatarsal amputation of the toe for the remaining $4 \%$ of cases (12 patients).

A percentage of $24 \%$ of patients ( 77 patients) have shown, upon admission to the hospital, a condition of extensive over-infected ischemic gangrene of the toe/s or foot, and for these patients a major amputation was required as a first surgical intervention.

A septic condition was present upon admission to the hospital in $53 \%$ of cases (170 patients), who needed antibiotics therapy, in many cases even requiring an association of two antibiotics ( $3^{\text {rd }}$ generation cephalosporin and metronidazole) and for the rest of the patients from the analyzed lot a prophylactic antibiotics therapy was administered.

The general mortality was of $2 \%$ (6 patients), of which 4 cases were associated with cardiovascular diseases in an advanced state of evolution and only 2 cases were associated with the septic conditions shown by the patient at the time of admission to the hospital, that was the cause leading to the evolution towards exitus.

\section{Discussions}

The diabetic foot treatment is difficult to manage, as it requires a multidisciplinary cooperation, an adequate antibiotic therapy, permanent education of the patient with respect to coming to the doctor as early as possible, at the time the lesions occurs. The surgical indication is given after obtaining the necessary investigation package, abiding by the general principles, but at the same time adapted to each individual case, therefore requiring long experience in the field. Sometimes, the generally altered state of health that the patient is in when coming to the hospital requires an emergency surgical intervention for a vital purpose, while in other instances the condition of the vascular bed allows for a surgical revascularization intervention, attempting to save the pelvic limb and practising an intervention to the focal point. [12]

As far as the use of surgical revascularization methods is concerned, it being a newly applied method in our clinic, we cannot make statistical statements about the results, especially about their evaluation on the long term, however, the first results indicate a large rate of success in saving the pelvic limb in patients with an anklebrachial index of less than 0.5 .

The surgical indication varies according to three categories of factors: the lesion type, the vascular status and the presence of infection. (table 1)

For patients admitted with ischemic gangrene lesions localized at the toe/s level, having an ankle-brachial index of less than 0.5 , and 93 of the total 298 showing signs of septicity that required a more extended intervention into healthy tissue, four types of surgical interventions were performed, depending on the extent of the necrosis process, namely: excisional debridement, for patients with limited, small size lesions, transphalangeal resection for patients that showed an ischemic necrosis process associated to a phalange, transmetatarsal amputation of the toe/s, for patients having lesions in one or more

Table 1 - Surgical indication in arteriopathic diabetic foot lesions

\begin{tabular}{lcc}
\hline Lesion Type & Vascularisation (ankle-brachial index) & Infection \\
\hline $\begin{array}{l}\text { Ischemic Gangrene of Toe } / \mathrm{s} \\
\text { - toe amputation }=147\end{array}$ & $27 \%$ between 0.5 and 0.8 & 93 patients \\
- transphalangeal $\mathrm{a} .=38$ & $73 \%$ less than 0.5 & \\
- excisional debridement $=54$ & & \\
- transmetatarsal $\mathrm{a} .=51$ & & 77 patients \\
Over-infected Gangrene, \\
extensive to toe/s, foot \\
- major amputation $=77$
\end{tabular}



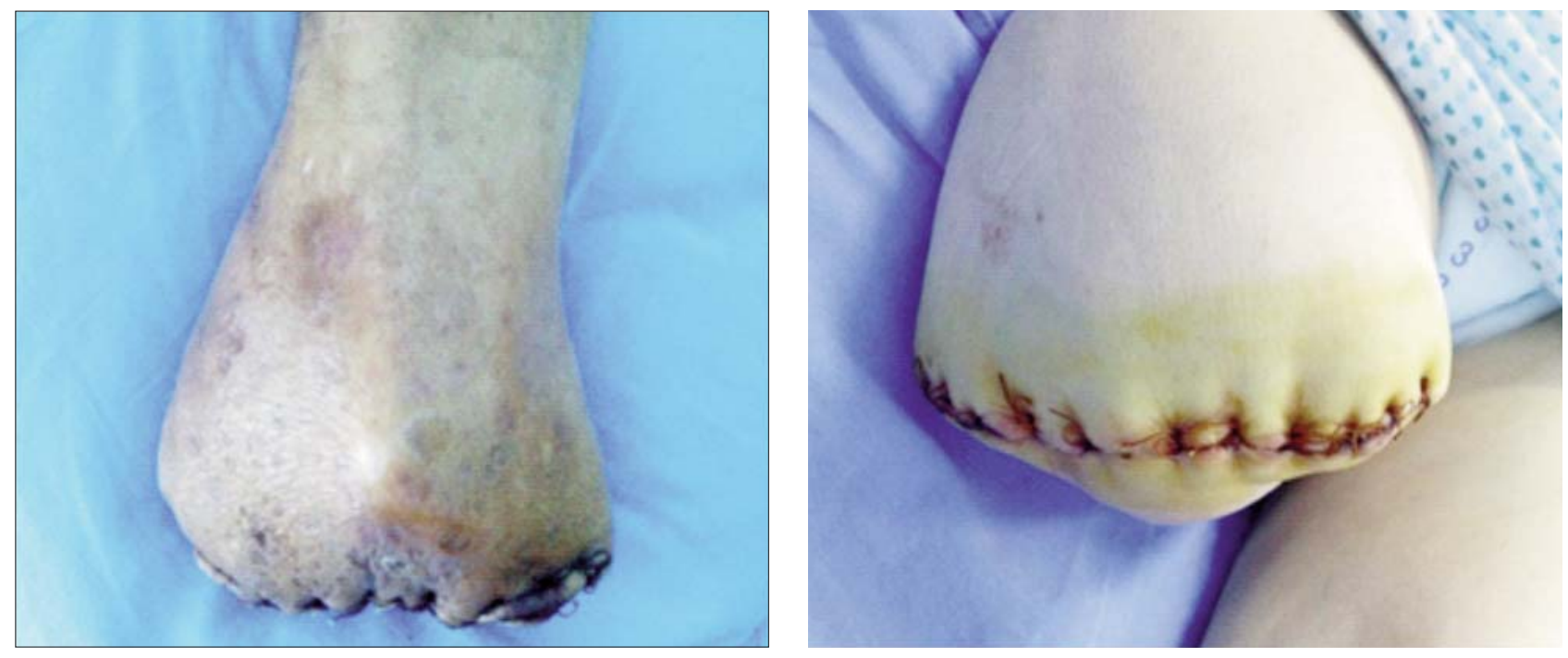

Figure 4 - amputation 1/3 average lower leg and 1/3 average upper leg

toes and transmetatarsal amputation of the foot for patients having lesions localized in at least four toes and/or showing an extension of the gangrenous process towards the forefoot.

For all patients with over-infected, extensive, toe/s or foot ischemic gangrene lesions, major amputations have been performed (on the lower leg and upper leg), as the patients were showing signs of septicity and an ankle-brachial index of less than 0.5 . For $7 \%$ ( 22 patients) of these patients, this surgical intervention has been a second major amputation. (fig. 4)

\section{Conclusions}

Prevention of complications of the diabetic foot is done, first and foremost, by educating patients about the possibility of occurrence of initially painless lesions, for which they must come and see a specialist, and they must also be made aware of the fact that they are predisposed to the occurrence of successive lesions.

The multidisciplinary treatment and prevention added to the correct surgical approach, are the most important issues in preserving the lower limb and fastening recovery. If a severe infectious process is also present, it will be difficult to balance the diabetic disease and this may lead to a decompensation of the other diseases of the patient, having an impact on their general health and requiring a complex therapeutic support.

Knowledge of the diabetic foot pathology is helpful in increasing the accuracy of the surgical indication, for the purpose of limiting the number of major amputations and applying an adequate prophylactic treatment, in view of bringing improvement in the quality of life of these patients.

Surgical indication for patients with diabetic foot lesions, with predominant arteriopathic terrain, is established when vascular surgery, contraindicate the use of revascularization, and if the infectious factor is associated, the lesion acquires immediate surgical treatment.

\section{Aknowledgement}

This paper is partly supported by the Sectorial Operational Programme Human Resources Development (SOPHRD), financed by the European Social Fund and the Romanien Government under the contract number POSDRU 159/1.5/S/141531.

\section{References}

1. Oxford: Handbook of endocrinology and diabetes

2. Pătrașcu T,Catrina E.,Doran H,Bugă C., Munteanu A., Serafinceanu C., "Particularities of Diabetic Surgery", Ed.Niculescu,2005. Cap1. 36-42

3. Golden SH, Peart Vigilance C, Kao WH, Brancati FL, "Perioperative glycemic control and the risk of infectious complications in a cohort of adults with diabetes" in Diabetes Care. 1999 Sep;22(9):1408-14

4. Băcanu Gheorghe, "Diabetic Gangrene”, Ed.Facla,Timișoara 1973;11-45,51-56

5. International Diabetes Federation. Diabetes and Foot care, Brussels 2005

6. Vereșiu I. . Diabetic foot Etiopathogenesis.Screening.Diagnosis.Treatment principles", Ed Echinox, 2005 
7. Morykwas, M.J., Argenta, L.C. "Nonsurgical modalities to enhance healing and care of soft tissue wounds", J. South Orthop. Assoc., 1997, 6:279

8. Prof. David G. Armstrong "Negative pressure wound therapy after partial diabetic foot amputation: a multicentre, randomised controlled trial" , The Lancet, Volume 366, Issue 9498, Pages 1704 - 1710, 2005

9. R.F. Tatu, R. Brediceanu, C. Ungurean, C. Cebzan, Carmen Tatu, L. Jiga, D. Vermesan, "Treatment of infected wounds by negative pressure terapy", Chirurgia, 102 (5): 577-580, 2003

10. Ubbink D.T., et al "Skin Microcirculation in Diabetic and NonDiabetic Patients and Different Stages of Lower Limb Ischaemia" European Jounal of Vascular Surgery 1993;7(6):659-6

11. Rubba P., et al „Diabetes Mellitus and Localisations of Obliterating Arterial Disease of the Lower Limbs", Angiology
1991;42(4):296-301.

12. Roger E Pecoraro, Gayle E Reiber and Ernest M Burgess „Pathways to Diabetic Limb Amputation: Basis for Prevention”, Diabetes Care May 1990 vol. 13, no. 5 513-521

13. Vascular Surgery Commission of the Romanian Ministry of Health „Management of Critical Ischemia of the Limbs and Diabetic Foot", Bucharest

14. Jeffcoate WJ "The incidence of amputation in diabetes", Acta Chir. Belg. 2005;105:140-144

15. American Diabetes Association: Clinical Practice Recomandations

16. Frykberg RG, Armstrong DG, Giurini J, et al. (2000). "Diabetic foot disorders: a clinical practice guideline. American College of Foot and Ankle Surgeons"

17. Majeski J.A., John J.F. Jr., " Necrotizing Soft Tissues Infections: A Guide to Early Diagnosis and Initial Therapy", South Med J 96(9):900-905,2003 